\title{
Segurança alimentar e nutricional: interfaces e diminuição de desigualdades sociais
}

\section{Food and nutrition security: interfaces and decrease of social inequality}

\author{
Cilene da Silva Gomes Ribeiro? \\ Maria Cecilia Barreto Amorim Pilla ${ }^{2}$ \\ ' Escola de Saúde e Biociências, Curso de \\ Nutrição. Pontifícia Universidade Católica do \\ Paraná, Curitiba-PR, Brasil \\ ${ }^{2}$ Escola de Educação e \\ Humanidades, Coordenação do Curso de História. \\ Pontifícia Universidade Católica do Paraná. \\ Curitiba-PR, Brasil \\ Correspondência / Correspondence \\ Cilene da Silva Gomes Ribeiro \\ E-mail: cilenex@hotmail.com
}

\section{Resumo}

Muitas mudanças têm ocorrido na forma da sociedade se alimentar ao longo da história. Com crescimento do processo de urbanização, o cotidiano das grandes cidades têm se caracterizado por práticas alimentares que priorizam a rapidez, economia e praticidade, muitas vezes em detrimento da qualidade, quantidade, do aspecto nutricional, social e ético que o alimento representa. Ao mesmo tempo em que muitos ainda não têm acesso ao alimento, situação particularmente resultado de desigualdades sociais, emerge um perfil alimentar caracterizado pelo consumo em excesso de alimentos industrializados, ricos em açúcares e gorduras, acarretando mudanças no perfil epidemiológico mundial e da população brasileira. O contraste das doenças causadas pelos excessos alimentares e a fome resulta em situações de insegurança alimentar e nutricional, gerando desumanização e aumento das desigualdades. Deve-se estudar todos os focos e enfoques que se relacionam, pois essa temática é fundamental no que tange a humanização, respeito e conduta ética do ser humano, dos governos e das comunidades.

Palavras-chave: Segurança alimentar e nutricional. Consumo. Consumo de Alimentos. Desigualdades. Iniquidade Social.

\section{Abstract}

Many changes have occurred in the form society is fed throughout history. With growing urbanization, everyday in big cities have been characterized by eating practices that prioritize speed, economy and practicality, often at the expense of quality, quantity, 
nutritional, social and ethical aspect that food represents. While many still lack access to food, particularly a result of social inequalities situation emerges a dietary pattern, characterized by excessive consumption of processed foods rich in sugar and fat, causing changes in the world and epidemiological profile of the population. The contrast between diseases caused by excessive food and hunger results in situations of food and nutrition insecurity, generating dehumanization and increasing inequalities. Studying all foci and related approaches is necessary, as this issue is critical when it concerns humanization, respect and ethical conduct of human beings, government and communities.

Key words: Food and Nutritional Security. Consumption. Food Consumption. Inequalities. Social Inequity.

\section{Introdução}

Desde a década de 1930, as ciências humanas assumiram formalmente seus interesses sobre o tema da alimentação. Segundo Burke, ${ }^{1}$ isso se deu com os fundadores dos Annales, os historiadores Marc Bloch e Lucien Fébvre.

Bloch escreveu um ensaio sobre história da alimentação, como parte integrante da obra Encyclopédie Française. Mais tarde, Fernand Braudel ${ }^{2}$ trouxe grandes contribuições sobre o tema, principalmente em seus estudos sobre a história da cultura material, ao estudar os gêneros alimentares, como o arroz e o trigo como produtos presentes no cotidiano europeu entre os séculos XV e XVIII. No Brasil, muito se tem avançado nos últimos anos em pesquisas em torno da temática alimentar em estudos de Antropologia, Sociologia e História.

A partir dessa premissa, o presente artigo pretende abordar as questões alimentares à luz da história e da cultura da alimentação, principalmente no que diz respeito à Segurança Alimentar e Nutricional em sua relação com interfaces políticas, econômicas e sociais. No que se refere ao tema, buscar dados referentes às práticas alimentares, bem como da produção de alimentos e do consumo, é fundamental para o entendimento da fome, da obesidade e do consumo. 


\section{Alimentação, nutrição e sociedade contemporânea}

Para Santos $^{3}$, a alimentação e nutrição referem-se tanto às práticas alimentares que são condicionadas pela produção, disponibilidade e acesso, quanto à forma como o organismo transforma e utiliza o alimento, e sua relação com a saúde.

As mudanças ocorridas na forma da sociedade se alimentar nas últimas décadas, no Ocidente, têm priorizado a rapidez, economia e praticidade, em detrimento muitas vezes da qualidade, quantidade e do aspecto social, cultural e ético que o alimento representa, gerando desumanização nas relações individuais e sociais e aumentando cada vez mais as desigualdades sociais. Nessa nova sociedade do trabalho, onde tempo é dinheiro, acentua-se a "irracionalidade" dos comportamentos alimentares, evidenciando-se uma grande relação entre a obesidade, novos rituais do ato de comer e o consumo de alimentos industrializados.

Associado a isso, surgiram situações de insegurança alimentar, caracterizadas por diferentes tipos de problemas, como fome, obesidade, doenças associadas à má alimentação e consumo de alimentos de qualidade duvidosa ou prejudicial à saúde, bem como a produção predatória de alimentos em relação ao meio ambiente, preços abusivos e a imposição de padrões alimentares que não respeitam a diversidade cultural.

Para Pipitone e Garcia $^{5}$, o contínuo processo de industrialização trouxe significativas mudanças na forma de se alimentar, especialmente no que diz respeito às grandes cidades. As transformações foram intensificadas com a profissionalização, independência das mulheres e com a Revolução Verde, que fez com que a alimentação "tradicional" perdesse espaço para novas práticas alimentares que simplificam o trabalho e economizam o tempo de preparo.

O paradoxo está posto. De um lado, encontra-se um perfil alimentar caracterizado por excessos no consumo de alimentos ricos em gorduras, açúcares e produtos industrializados com alta densidade energética; de outro, a fome e a desnutrição, geradas pela indisponibilidade de alimentos, pela escassez, ou pela falta de uma educação alimentar esclarecedora no sentido nutricional.

Almeida ${ }^{6}$ e Levy-Costa ${ }^{7}$ consideram que o modelo predominante de produção de alimentos também tem favorecido o paradoxo alimentar dessa nova sociedade, afinal a disponibilidade de produtos industrializados uniformiza práticas alimentares com refeições rápidas e ricas em alimentos processados e pré-preparados, gerando muitas vezes uma fome oculta e determinando a alta prevalência de doenças crônicas não transmissíveis, tais como a obesidade, hipertensão, diabetes do tipo II e dislipidemias. 
Segundo documento do Conselho Nacional de Segurança Alimentar e Nutricional (CONSEA) ${ }^{8}$, o contraste entra as doenças causadas pelos excessos alimentares e a fome resulta em situações de insegurança alimentar e nutricional. As vantagens nutricionais não estão relacionadas exclusivamente aos nutrientes; também estão relacionadas com a Segurança Alimentar e Nutricional (SAN). Esta contempla, além da aquisição regular e permanente do alimento, de qualidade e em quantidade suficiente, as bases práticas alimentares promotoras da saúde, que respeitem a diversidade cultural e que sejam ambiental, econômica e socialmente sustentáveis.

O conceito de Segurança Alimentar veio à luz a partir da Segunda Grande Guerra, diante de uma Europa devastada e sem condições de produzir seu próprio alimento. Segundo Belik ${ }^{9}$, esse conceito foi construído levando-se em conta três aspectos principais: quantidade, qualidade e regularidade no acesso e de disponibilidade de alimentos. Assim, por exemplo, pode ser que haja grandes quantidades de alimentos sendo produzidos em determinada região, mas se as populações não podem não ter acesso a eles, a situação de Segurança Alimentar não se configura.

Da mesma maneira, em relação à qualidade, especialmente, a alimentação disponível para o consumo da população não pode estar submetida a qualquer tipo de risco por contaminação, problemas de apodrecimento ou outros decorrentes de prazos de validade vencidos. A qualidade dos alimentos diz respeito também à possibilidade de consumi-los de forma digna. Ou seja, permitir que as pessoas possam comer em um ambiente limpo, e seguindo as normas tradicionais de higiene.

No que se refere à definição de Segurança Alimentar, é preciso que não se deixe de abordar a questão da regularidade. Isso quer dizer que as pessoas têm que ter acesso constante à alimentação (alimentando-se ao menos três vezes ao dia). De acordo com Pessanha ${ }^{10}$, identificar e avaliar este quesito é fundamental, dentro das abordagens éticas - afinal, não se considera isenta de risco uma população que não tenha acesso aos alimentos nesse sentido.

Em nossa abordagem, não poderemos deixar de citar também a soberania e sustentabilidade alimentar. O emprego da noção de soberania alimentar começa a surgir com força no debate do tema da Segurança Alimentar, em 1996. Durante a Cúpula Mundial da Alimentação, no foro paralelo da sociedade civil realizado em Roma, a reivindicação da soberania alimentar apareceu com grande destaque. Esse conceito procura dar importância à autonomia alimentar dos países e está associado à geração de emprego e à menor dependência das importações e flutuações de preços do mercado internacional. A soberania alimentar atribui grande importância à preservação da cultura e aos hábitos alimentares de um país, por isso deve ser abordada de forma ética e comprometida, conforme Belik ${ }^{9}$. Incorpora conceitos ligados à preservação do meio ambiente, a não utilização de agrotóxicos e contrários à produção extensiva em monoculturas. Aqui é possível avaliar os efeitos e percepções dos alimentos transgênicos e de todas as culturas tecnicistas.

Segundo o mesmo autor, a utilização do conceito de Segurança Alimentar dá origem a diferentes interpretações e não se pode ignorar a importância das políticas em torno desse tema, 
como mobilizadoras das forças produtivas. Quanto à oferta, a Segurança Alimentar pressupõe a garantia de que haja disponibilidade suficiente e sustentável de alimentos que respeitem a cultura alimentar, que sejam física e economicamente acessíveis à população e que propiciem opções saudáveis de alimentação. Isso requer, entre outros aspectos, o uso criterioso e sustentável dos recursos naturais da sociedade, o emprego de tecnologias seguras e eficientes e a execução de políticas governamentais que estimulem a produção e comercialização de alimentos saudáveis, não dispendiosos e compatíveis com a cultura alimentar local.

Do lado da demanda, a Segurança Alimentar pressupõe a garantia de que todos os indivíduos saberão identificar e terão capacidade de adotar opções saudáveis de alimentação. Isso demanda, entre outros aspectos, níveis mínimos de renda (ou capacidade de autoconsumo) da população e acesso universal a conhecimentos básicos sobre a relação entre alimentação e saúde, composição nutricional dos alimentos e recomendações dietéticas.

O acesso a conhecimentos é particularmente relevante em sociedades onde os padrões tradicionais de alimentação não são inteiramente saudáveis e/ou onde padrões tradicionais saudáveis de alimentação estejam sendo substituídos por padrões menos saudáveis, seja por mudanças na estrutura de preços dos alimentos e/ou no nível de renda da população, seja por força de estratégias agressivas de propaganda de alimentos não saudáveis.

O estudo e o monitoramento da Segurança Alimentar de uma sociedade são complexos, uma vez que são vários os elementos que a definem. Do lado da oferta de alimentos, devem ser estudados e monitorados, no mínimo, a disponibilidade per capita, o tipo, a composição nutricional, a qualidade sanitária, a obediência a regulamentações legais (eventuais prazos de validade e rotulagem nutricional), a cobertura da comercialização e o preço dos alimentos ofertados para consumo. No caso de alimentos não saudáveis, importa igualmente monitorar a quantidade produzida, estratégias de promoção e obediência a eventuais limitações legais na propaganda desses alimentos. Do lado da demanda, devem ser estudados e monitorados a renda, a capacidade de autoconsumo, as preferências alimentares, os conhecimentos sobre alimentação, nutrição e saúde e os padrões de consumo alimentar da população.

Enfim, é fundamental que cada um desses elementos seja avaliado criteriosa e individualmente, pois as ações necessárias para promover as condições de segurança alimentar da sociedade, gerar humanização e reduzir desigualdades serão distintas conforme os problemas encontrados.

Diante do processo de transição nutricional, caracterizado pelo aumento do excesso de peso em detrimento da desnutrição, observado em adultos, crianças e adolescentes, e de suas consequências negativas nos estágios futuros da vida, torna-se importante o monitoramento da situação alimentar e nutricional da população, a partir do enfoque da humanização e da redução das desigualdades. 


\section{Comer?}

A alimentação, por atender a uma das necessidades básicas do homem, a sobrevivência, sempre foi objeto de preocupação individual e coletiva. E sendo também uma forma de expressão dos desejos humanos, o alimento não deve ser apenas um objeto que fornece nutrientes, mas deve dar prazer e possuir um prestígio, um valor que lembra conforto, como bem concordam Sloan, ${ }^{11}$ Carneiro $^{12}$ e Poulain. ${ }^{13}$

Deste modo, no Brasil, a realização do direito à alimentação - promulgada na Emenda Constitucional 64, que incluiu a alimentação entre os direitos sociais, fixados no artigo $6^{\circ}$ da Constituição Federal, no dia 05 de fevereiro de 2010 - é exposta por meio da Segurança Alimentar e Nutricional (SAN). Esta visa garantir o direito de todos ao acesso regular e permanente a alimentos de qualidade, em quantidade suficiente, sem comprometer o acesso a outras necessidades essenciais. Tem como base práticas alimentares promotoras da saúde, que respeitem a diversidade cultural e que sejam ambiental, cultural, econômica e socialmente sustentáveis, como bem prevê o CONSEA. ${ }^{14}$

A SAN está intimamente ligada a uma política pública, por meio do CONSEA - realizado entre o governo e a sociedade como instrumento de articulação para assessorar a Presidência da República na formulação de políticas e na definição de orientações para que o país garanta o direito humano à alimentação. O objetivo é fazer cumprir o dever do poder público de respeitar, proteger, promover, prover, informar, monitorar, fiscalizar e avaliar a realização do direito humano à alimentação adequada, bem como garantir os mecanismos para sua exigibilidade. E é através dos princípios de abrangência, intersetorialidade, equidade, participação social e articulação entre medidas de caráter emergencial e estrutural, bem como as sete diretrizes, que a SAN impõe uma política pública, também conhecida como Política Nacional de Segurança Alimentar e Nutricional, prevista pelo CONSEA em 2009.15

No entanto, o comportamento referente à comida vai além do biológico e nutricional, pois a alimentação é repleta de aspectos históricos, sociais, culturais e psicológicos. Para Santos, ${ }^{3}$ "alimentar-se é um ato nutricional, comer é um ato social, pois constitui atitudes ligadas aos usos, costumes, protocolos, condutas e situações", fazendo com que o alimento não seja neutro. Essa transmissão das estruturas culturais da alimentação se dá desde a infância, não sendo necessariamente realizada pelo ensinamento direto dos pais para os filhos.

A história da alimentação abrange, mais do que a história dos alimentos, sua produção, distribuição, preparo e consumo. O que se come é tão importante quanto, quando, onde, como e com quem se come. A refeição em família é um ritual propício à transmissão de valores, por meio da aprendizagem de maneiras, sobretudo as de mesa. É quando se desenham, para a criança, os contornos do mundo ao qual ela pertence, e as atitudes aprovadas por seu grupo social são 
assimiladas como norma, fazendo com que os homens comam como a sociedade os ensinou. Assim os hábitos alimentares se tornam simultaneamente resistentes e abertos a mudanças, pois essa transformação acompanha o dinamismo da sociedade, hábitos inicialmente transmitidos pela família, através do contato com outras culturas; padrões de consumo são acrescidos de novas práticas alimentares, conforme preveem Franco ${ }^{16}$ e Pineyrua. ${ }^{17}$

Formados também a partir da disponibilidade local de produtos, os hábitos e costumes alimentares representam o simbolismo e a lógica de suas práticas, dos valores familiares e das condições e relações sociais de grupos, povos e nações. Afirmando-se como identidade cultural, cada um desses aspectos determina a qualidade, a apresentação e os horários da comida. Para Carneiro, ${ }^{18}$ partilhar o alimento tem significados que demonstram civilidade; comer é a origem da socialização. A oferta de determinado alimento em vez de outro nunca é casual, mas reproduz as relações de poder e prestígio.

Há alguns exemplos significativos já nos grandes poemas gregos que narram ações grandiosas e heroicas, como por exemplo, os poemas homéricos. Nessa literatura, existem referências à oferta dos melhores pedaços aos hóspedes. Outro exemplo nos traz Montanari, ${ }^{19}$ segundo o qual, na literatura céltica, são narradas lutas furiosas entre os chefes de tribos antagonistas ou clãs rivais para obtenção dos melhores pedaços de carne.

\section{Urbanização e alimentação}

Com a mudança dos modos de vida, nos anos 1950-1960, gerados pela industrialização e urbanização, como nos dizem Flandrin \& Montanari, ${ }^{20}$ ocorre o deslocamento maciço das populações rurais para as cidades, implicando custos de produção bastante elevados da mão de obra urbana, em particular por causa das dificuldades encontradas no abastecimento alimentar das regiões comerciais, bem como dos centros industriais.

A industrialização também proporcionou a Revolução Verde, fundamentada na produção alimentar de larga escala com alta tecnologia, buscando a alta produtividade para suprir as regiões com predomínio da fome. $\mathrm{O}$ aumento da produtividade, a maior resistência às doenças e às pragas, o decréscimo no tempo necessário para produzir e distribuir novos cultivos de plantas maximizaram o empobrecimento dos recursos naturais, juntamente com a perda da biodiversidade, além de abrir um novo e vasto mercado para as operações das grandes multinacionais produtoras de sementes, fertilizantes e pesticidas. Segundo Cavalli, ${ }^{21}$ podem também ter contribuído também para uma série de novos riscos à saúde, como o aumento das alergias e resistência aos antibióticos.

É preciso considerar também, como bem nos alertam Flandrin e Montanari, ${ }^{20}$ que a partir das décadas de 1960 e 1970, devido, entre outros fatores, à presença mais constante da mulher no mercado de trabalho, diminui-se a disponibilidade de tempo para o preparo das refeições 
da família. Deste modo, esse conjunto de acontecimentos - industrialização, urbanização, independência das mulheres, profissionalização, aumento da variedade e acesso agroalimentar estabelecido pela Revolução Verde - gerou a desregulação das práticas alimentares e aumento do número de refeições tomadas fora de casa, na empresa, escola e coletividades.

Pondera Guivant ${ }^{22}$ que as tradicionais lojas de produtos naturais, feiras e açougues passaram a ter papel secundário a partir de então. Surgiu a preferência pelos supermercados para a compra dos alimentos, movidos pela busca de praticidade e economia de tempo. Com isso, as empresas de fast food e similares, caracterizadas pela capacidade de preparar e servir refeições em curto intervalo de tempo aumentaram em número. Para Castelli, ${ }^{23}$ o espírito da "McDonaldização" levou essas empresas ao máximo de produtividade e eficiência, atendendo às necessidades da sociedade atual.

Concomitantemente à aceleração no ritmo de vida e a ascensão do fast food, afirmam Miele \& Murdoch ${ }^{24}$ que houve o declínio no conhecimento sobre a culinária tradicional e a preferência alimentar da maioria das pessoas mudou dos pratos regionais e locais para os internacionais. Nesse sentido, para Oliveira, ${ }^{25}$ a mudança no padrão alimentar, sobretudo como consequência das políticas que favoreceram a opção pelo modelo de desenvolvimento econômico do tipo capitalista, levou à criação de novos padrões de produção e de consumo.

O diagnóstico efetuado pelo CONSEA ${ }^{15}$ apontou para a confluência de quatro crises sistêmicas (econômica, alimentar, ambiental e energética) que estão inter-relacionadas em suas principais causas e impactos e requerem respostas igualmente sistêmicas e coordenadas. A crise econômica contribuiu para limitar significativamente a capacidade de acesso aos alimentos dos mais pobres e ampliou o número de famintos no mundo, além de repercutir sobre o sistema de crédito e a disponibilidade de recursos para os programas sociais e de apoio à agricultura familiar e camponesa. As crises econômica, energética e alimentar afetaram negativamente as tendências de redução da pobreza e da desigualdade que se verificam há alguns anos em muitos países da América Latina e do Caribe, onde voltou a ocorrer aumento no número de famintos em 2009.

Os impactos domésticos da crise revelaram o elevado grau de exposição externa dos países da região, apesar de serem grandes produtores de alimentos. Nas crises alimentar e econômica encontram-se evidências claras da falência das teses do Estado-mínimo e da desregulamentação das atividades econômicas que dominaram a cena econômica e política nas últimas décadas. Do mesmo modo, as problemáticas ambiental e energética têm entre suas causas a ausência de regulação sobre o uso de recursos e suas repercussões sobre bens comuns ou públicos. A fragilidade dos países em desenvolvimento como o Brasil que, até recentemente, optaram pelo caminho da liberalização comercial e da desregulamentação econômica contrasta com o ressurgimento de um padrão no qual ganharam, novamente, importância os Estados nacionais (ou blocos regionais como a União Europeia) com os respectivos instrumentos de regulação dos mercados agroalimentares. 
Soma-se a isso o quadro epidemiológico e nutricional do país, onde se convive com números, cada vez mais crescentes, de doenças e mortes relacionadas à má qualidade da alimentação. Além disso, persistem quadros de carências de vitaminas e minerais e de desnutrição em grupos populacionais vulnerabilizados, que atingem de maneira diferenciada, mas igualmente significativa, todos os grupos etários, extratos de renda e regiões. Paradoxalmente aos dados indicativos de desnutrição no país, o sobrepeso e a obesidade, assim como as doenças não transmissíveis delas decorrentes, passaram a compor o quadro da saúde pública no Brasil, sendo frequentes também em populações de baixa renda. Diante disso, percebe-se que o perfil alimentar reproduz um padrão globalizante de oferta de alimentos com baixo custo, mas deficientes em qualidade nutricional.

Em conformidade com os estudos de Caballero \& Popkin, ${ }^{26}$ atualmente a sociedade brasileira, junto com o resto do mundo, vive um período chamado de transição nutricional, em que, apesar da fome e da desnutrição ainda estarem presentes, são crescentes as prevalências de excesso de peso e das doenças crônicas devido a mudanças no padrão alimentar e de atividade física.

A alimentação adequada, nos aspectos de qualidade e quantidade, fornece ao organismo energia e nutrientes necessários para o perfeito desenvolvimento de suas funções e manutenção da saúde. E o contrário constitui fator de risco para desnutrição e carência de micronutrientes. Por outro lado, o excesso alimentar relaciona-se à ocorrência da obesidade e de suas comorbidades, como diabetes, hipertensão arterial, problemas cardiovasculares, entre outros.

\section{Políticas Públicas de Segurança Alimentar e Nutricional}

O governo federal, a partir das Políticas Nacionais de Segurança Alimentar e Nutricional, tem tentado promover um conjunto de ações planejadas que garantam a oferta e o acesso aos alimentos para toda a população, promovendo a nutrição e a saúde. ${ }^{27}$ O CONSEA, por meio das suas sete diretrizes, estabelece o envolvimento tanto do governo quanto da sociedade civil organizada, nos diferentes setores ou áreas de ação - saúde, educação, trabalho, agricultura, desenvolvimento social, meio ambiente, dentre outros - e em diferentes esferas - produção, comercialização, controle de qualidade, acesso e consumo. Afinal, Segurança Alimentar e Nutricional consiste em garantir a todos de condições de acesso a alimentos de qualidade em quantidade suficiente, de modo permanente e sem comprometer o acesso a outras necessidades essenciais, com base em práticas alimentares saudáveis, contribuindo assim para uma existência digna.

A política de SAN avança em relação às ações e programas desenvolvidos pelos diferentes segmentos, ao promover os princípios de intersetorialidade, ações conjuntas entre Estado e sociedade; equidade, superando as desigualdades econômicas, sociais, de gênero e étnicas que ainda hoje geram discriminações sobretudo contra negros e indígenas; abrangência e articulação entre ações estruturantes e medidas emergenciais e orçamento e gestão. 
Segundo o mesmo documento do CONSEA, ${ }^{27}$ a SAN também atua sobre as esferas da produção e disponibilidade de alimentos; do acesso ao alimento e do abastecimento; da alimentação e nutrição. Essas dimensões expressam os dois componentes básicos de uma política de SAN: o componente alimentar (relacionado com a disponibilidade, produção, comercialização e acesso ao alimento) e o componente nutricional (relacionado às práticas alimentares: onde, como e quando se alimenta, o que se escolhe, como se prepara e o que se consome, e à utilização biológica do alimento).

É através de diretrizes voltadas para uma alimentação de qualidade - alimentos seguros, saudáveis, que não gerem doenças e promovam a qualidade de vida, em quantidades suficientes sem comprometer o acesso a outras necessidades essenciais; junto ao direito a saúde, educação e trabalho; tendo como base práticas alimentares que promovam a saúde, direito a informação, rotulagem explícita e segura; que respeitem a diversidade cultural e que sejam ambiental, cultural, econômica e socialmente sustentáveis, representando o direito de preservação do alimento como patrimônio - que ocorrerão a humanização e a redução das desigualdades.

É através do fortalecimento da agricultura familiar, da busca pela sustentabilidade, da recuperação do convívio durante as refeições, do respeito pela biodiversidade, da preservação do patrimônio gustativo, da promoção de práticas alimentares em crianças que se reforçam estratégias na busca pela Segurança Alimentar e Nutricional no Brasil e no mundo.

Como parte integrante dos avanços obtidos no enfrentamento da pobreza e da desigualdade social no país, o acesso aos alimentos se ampliou nas camadas de menor renda da população, como resultado das políticas públicas de expansão do emprego formal e de recuperação do poder de compra do salário mínimo e suas repercussões sobre os benefícios da seguridade e assistência social, e da ampla cobertura de transferência de renda propiciada pelo Programa Bolsa Família.

Como exemplos de construções intersetoriais, sobressaem o Programa de Aquisição de Alimentos da Agricultura Familiar (PAA) e a recente reformulação do Programa Nacional de Alimentação Escolar (PNAE). Mencione-se também a perspectiva de articular equipamentos públicos de alimentação em sistemas locais (restaurantes populares, bancos de alimentos, cozinhas comunitárias e outros) e ampliar e qualificar as ações de alimentação e nutrição no nível de atenção primária em saúde.

\section{Considerações Finais}

Diante da transição nutricional, torna-se cada vez mais importante e necessária a pesquisa acadêmica como instrumento de vigilância alimentar e nutricional para monitoramento e planejamento de ações de saúde, garantindo bases teóricas para a análise da fome, da obesidade, do acesso limitado e do ilimitado consumo, das desigualdades geradas pela carência e pelo excesso, da humanização e da desumanização dos atos de alimentar. 
O diálogo multidisciplinar entre a nutrição, a sociologia e a história se fortalece neste tema, uma vez que o homem é o que come e, através do que come, desenvolve o local onde vive. Estabelecer interseções entre os contextos políticos, econômicos, ambientais, sociais e históricos é fundamental para que a evolução da nutrição seja compreendida e assim, seja possível reduzir as inúmeras desigualdades existentes.

\section{Referências}

1. Burke P. A escola dos Annales. São Paulo: Unesp; 1991.

2. Braudel F. Civilização material, economia e capitalismo, séculos XV-XVIII. As estruturas do cotidiano. São Paulo: Martins Fontes; 2005.

3. Santos CRA. O império McDonald e a McDonalização da sociedade: alimentação, cultura e poder. Seminário facetas do império na história. 6-10 nov. 2006; Curitiba, Brasil. Curitiba: UFPR; 2006. Disponível em: http://people.ufpr.br/ andreadore/antunes.pdf.

4. Pipitone MAP. Educação para o consumo de alimentos. Hig. Aliment. 2005; 19(132):18-23.

5. Garcia RWD. Reflexos da globalização na cultura alimentar: considerações sobre as mudanças na alimentação urbana. Rev. Nutr. 2003; 16(4):483-492.

6. Almeida SS, Nascimento PCBD, Quaioti TCB. Quantidade e qualidade de produtos alimentícios anunciados na televisão brasileira. Rev. Saúde Pública. 2002; 36(3):353-355.

7. Levy-Costa RB. Disponibilidade domiciliar de alimentos no Brasil: distribuição e evolução (19742003). Rev. Saúde Pública. 2005; 39(4):530-540.

8. Brasil. Conselho Nacional de Segurança Alimentar e Nutricional. A segurança alimentar e nutricional e o direito humano à alimentação adequada no Brasil. Brasília: CONSEA; 2010. Disponível em: http://www2.planalto.gov.br/consea/biblioteca/publicacoes/a-seguranca-alimentar-e-nutricionale-o-direito-humano-a-alimentacao-adequada-no-brasil

9. Belik W. Perspectivas para segurança alimentar e nutricional no Brasil. Saúde Soc. 2003; 12(1):12-20.

10. Pessanha LDR. Experiência brasileira em políticas públicas para a garantia do direito ao alimento. Rio de Janeiro: IBGE; 2002.

11. Sloan D. Gastronomia, restaurantes e comportamento do consumidor. Barueri: Manole; 2005.

12. Carneiro HS. Comida e sociedade: uma história da alimentação. Rio de Janeiro: Elsevier; 2003.

13. Poulain JP. Sociologias da alimentação: os comedores e o espaço social alimentar. Florianópolis: UFSC; 2004.

14. Brasil. Conselho Nacional de Segurança Alimentar e Nutricional. A segurança alimentar e nutricional e o direito humano à alimentação adequada no Brasil. Brasília: CONSEA; 2010. Disponível em: http://www2.planalto.gov.br/consea/biblioteca/publicacoes/a-seguranca-alimentar-e-nutricionale-o-direito-humano-a-alimentacao-adequada-no-brasil 
15. Brasil. Conselho Nacional de Segurança Alimentar e Nutricional. Construção do sistema e da política nacional de segurança alimentar e nutricional: a experiência brasileira. Brasília: CONSEA; 2009. Disponível em: http://www2.planalto.gov.br/consea/biblioteca/publicacoes/copy_of_versaoem-portugues

16. Franco A. De caçador a gourmet: uma história da gastronomia. 4. ed. São Paulo: Senac; 2006.

17. Pineyrua DGF. Regionalismo alimentar: identificação de grupos de consumidores que valorizam o prazer e as tradições alimentares. Campo Grande: UNB; 2006.

18. Carneiro HS. Comida e sociedade: significados sociais na história da alimentação. História: Questões \& Debates. 2005; 42:71-80.

19. Montanari M. Comida como cultura. São Paulo: SENAC; 2008.

20. Flandrin JL, Montanari M. História da alimentação. São Paulo: Estação Liberdade; 1998.

21. Cavalli SB. Segurança alimentar: a abordagem dos alimentos transgênicos. Rev. Nutr. 2001;14:41-46.

22. Guivant JS. Os supermercados na oferta de alimentos orgânicos: apelando ao estilo de vida ego-trip. Ambiente \& Sociedade. 2003; 6(2):63-81.

23. Castelli G. Gestão hoteleira. São Paulo: Saraiva; 2006.

24. Miele M, Murdoch J. The practical aesthetics of traditional cuisines: slow food in Tuscany. Sociol. Rural. 2002; 42(4):312-328.

25. Oliveira SP. Estudo do consumo alimentar: em busca de uma abordagem multidisciplinar. Rev. Saúde Pública. 1997; 31(2):201-208.

26. Caballero B, Popkin BM. The nutrition transition: diet and disease in the developing world. Maryland: Johns Hopkins University; 2002.

27. Brasil. Conselho Nacional de Segurança Alimentar e Nutricional. Princípios e diretrizes de uma política de segurança alimentar e nutricional. Brasília: CONSEA; 2004. Disponível em: http:// www2.planalto.gov.br/consea/biblioteca/publicacoes/principios-e-diretrizes-de-uma-politica-de-san

Recebido: 29/6/2013

Revisado: 06/9/2013

Aprovado: 30/11/2013 